\title{
A method for the treatment of supergranulation advection by giant cells (Research Note)
}

\author{
P. E. Williams` and M. Cuntz
}

\author{
Department of Physics, University of Texas at Arlington, Science Hall, Arlington, TX 76019, USA \\ e-mail: peter.williams@nasa.gov; cuntz@uta.edu \\ Received 10 December 2008 / Accepted 10 August 2009
}

\section{ABSTRACT}

\begin{abstract}
Aims. We present a new method for the treatment of the advection of solar supergranulation by giant cells, a large-scale analogue to the observed property of granule advection by supergranules.

Methods. The proposed method is derived from a description of solar convection via spherical harmonics and spectral coefficients, allowing the investigation of the influence of a giant cell component on a realistic supergranule signal.

Results. We show that a supergranule pattern derived from real data, as well as a simplified test signal, can be advected by a giant cell component of various sizes.

Conclusions. The identified behaviour is in analogy to observed supergranulation patterns, including those based on MDI Dopplergrams, which show wavelike supergranulation patterns, even after the removal of the geometric projection effect. Our method is an important step towards the construction of future models involving supergranule flow patterns advected by a giant cell flow. Nevertheless, additional efforts are required to obtain a final verification of giant cells as a separate component of the solar photospheric convection spectrum.
\end{abstract}

Key words. convection - methods: numerical - Sun: helioseismology - Sun: photosphere - turbulence - waves

\section{Introduction}

The different components of solar convection are well seen at the solar surface despite requiring their own methods of observation. Intensity and Doppler observations of the surface reveal bright cellular granule patterns that evolve over time. This granulation ( $\sim 1 \mathrm{Mm}$ across, $\sim 1 \mathrm{~h}$ lifetime) is considered to be convective flow driven by the recombination of atomic hydrogen below the photosphere. Supergranulation ( $\sim 30 \mathrm{Mm}$ across, $\sim 24 \mathrm{~h}$ lifetime), however, is not as readily observable as their smaller counterparts and requires Doppler measurements of the solar surface for it to be seen. The process of producing supergranules is not yet understood as those previously proposed such as helium recombination (Leighton et al. 1962) or accumulated downdrafts of granules (Rast 2003) seem not to create an independent supergranule component when scrutinized by numerical simulations (Stein \& Nordlund 1998; Stein 2008, private communication), although granules with a spatial size comparable to supergranules are found.

Mesogranules were claimed to exist as an independent convection component with size scales located between granules and supergranules (November et al. 1981). However, from studying the distribution of cell sizes using a photospheric convection spectrum derived from MDI Doppler maps (Hathaway et al. 2000), no exclusive mesogranule component seems to exist. Signals that are found at sizes typical of mesogranules $(\sim 7 \mathrm{Mm})$ are more appropriately ascribed to large granules or small supergranules.

^ New address: NASA Goddard Space Flight Center, Greenbelt, MD 20771, USA.
The largest component of convection, giant cells, still remain an enigma. Their existence was proposed by Simon \& Weiss (1968) after models predicted the production of cells $\sim 300 \mathrm{Mm}$ across with lifetimes of $\sim 34$ days. Physical evidence for these features, however, has been sparse and circumstantial at best. Observational techniques have been proposed and executed, but with no definitive results (Strous \& Simon 1998; Kitai et al. 1999). Other implicit signals may, however, betray the existence of giant cells (Beck et al. 1998; Ulrich 2001). Despite this lack of solid observational evidence of giant cells, extensive modelling has been performed (Miesch et al. 2007).

Previous observations of supergranules have revealed a large array of properties, including (1) their size, shape and their coincidence with the chromospheric network (Simon \& Leighton 1964; Küveler 1983); (2) their flow speeds (Küveler 1983; Hathaway et al. 2002); and (3) their advection of granules (Rieutord et al. 2008). Their Doppler signal also exhibits a superrotation characteristic with respect to the underlying plasma Duvall (1980). Hathaway et al. (2006) were able to attribute the majority of the signal to a geometric projection effect, but a remaining signal, including a retrograde component (Gizon et al. 2003), was still unaccounted for. Besides these extra longitudinal velocity signals, Schou (2003) detects northbound and southbound wave propagation at $40^{\circ}$ latitude that may correspond to poleward and equatorward flow signals, providing further tentative evidence of giant cells.

Future efforts to verify the existence of giant cells will require detailed simulations of the advection of supergranules by giant cell flows. This is the underlying motivation for the development of our method described in Sect. 2. In Sect. 3 we provide a summary of the work and a brief outlook. 


\section{Methods and results}

\subsection{Background}

Both previous and envisioned data simulations are aimed at obtaining insight into the scales and dynamics of solar photospheric convection. These types of simulations are not hydrodynamic nor MHD numerical simulations but rather simulations of the Doppler data itself in the form of vector velocities over the full solar surface that are projected onto the line-of-sight to reproduce the Doppler data characteristics (e.g., Hathaway et al. 2000, 2002).

In general, any vector velocity field on a spherical surface can be represented in terms of a radial component, a solenoidal (or poloidal) component (curl-free horizontal flow), and a toroidal component (divergence-free horizontal flow) with

$$
\begin{aligned}
& U_{r}(\theta, \phi)=\sum_{\ell=0}^{\ell_{\max }} \sum_{m=0}^{\ell} R_{\ell}^{m} Y_{\ell}^{m}(\theta, \phi) \\
& U_{\theta}(\theta, \phi)=\sum_{\ell=0}^{\ell_{\max }} \sum_{m=0}^{\ell}\left[S_{\ell}^{m} \frac{\partial Y_{l}^{m}}{\partial \theta}+T_{\ell}^{m} \frac{1}{\sin \theta} \frac{\partial Y_{l}^{m}}{\partial \phi}\right] \\
& U_{\phi}(\theta, \phi)=\sum_{\ell=0}^{\ell_{\max }} \sum_{m=0}^{\ell}\left[S_{\ell}^{m} \frac{1}{\sin \theta} \frac{\partial Y_{l}^{m}}{\partial \phi}-T_{\ell}^{m} \frac{\partial Y_{l}^{m}}{\partial \theta}\right]
\end{aligned}
$$

where the complex coefficients $R_{\ell}^{m}, S_{\ell}^{m}$, and $T_{\ell}^{m}$ give the radial, solenoidal, and toroidal parts (Chandrasekhar 1961), the $Y_{\ell}^{m}$ are the spherical-harmonic functions, and $R_{\ell}^{m}$ and $S_{\ell}^{m}$ are related through the mass continuity equation. Each of these four quantities are described over a range of spherical-harmonic degrees $\ell$ and azimuthal orders $m$. The angular quantities $\theta$ and $\phi$ measure the colatitude southward from the north pole and the azimuth prograde from the central meridian, respectively. Given input values for these complex spectral coefficients, vector velocities can be computed and projected onto the line-of-sight to produce Doppler velocity images (Hathaway 1988b).

The values for the spectral coefficients are obtained by modelling convection spectra obtained from full-disk solar observations made by the MDI instrument (Scherrer et al. 1995) aboard the SOHO spacecraft. The spectra show power (kinetic energy) per wavenumber for a large range of spherical harmonic degrees $\ell$ (Hathaway et al. 2000). Models are constructed from a two-component spectrum representing supergranules (with $\ell \sim$ 110 ) and granules (with $\ell \sim 4000$ ) using two broad Lorentzian profiles for the spectral amplitudes along with random phases to match the observations. This method has been used to create spectral coefficients used as input in recent studies (e.g., Hathaway et al. 2006; Williams et al. 2007) and continues to be used in the further development of the previously described data simulations (Hathaway et al. 2008).

\subsection{Advection of supergranulation by a giant cell component}

Next we describe how to consider the advection of supergranulation by a giant cell component. To study how flows of different length scales may interact, we formulate the following time-dependent approach. To begin with, a simplified synthetic flow pattern comprising of a single sinusoidal component at a wavenumber $m=100$ is constructed in a one-dimensional domain. Our aim is to advect this flow pattern signal with an underlying component constructed at a wavenumber $m=20$.

The advection is carried out by evolving the amplitude and phase information of the small-scale pattern. To synthesize this advection, we apply a one-dimensional version of the momentum equation given by

$\frac{\partial u_{\phi}}{\partial t}=-\frac{v_{\phi}}{R_{\odot}} \frac{\partial u_{\phi}}{\partial \phi}$

where $u_{\phi}$ denotes the supergranule signal and $v_{\phi}$ denotes the giant cell signal, while the derivatives are taken with respect to time $t$ and longitude $\phi . R_{\odot}$ is the solar radius.

Using a sinusoidal distribution for the one-dimensional poloidal flows along the equator only, $u_{\phi}$ is given as

$u_{\phi}=\sum_{m} S_{m} \mathrm{e}^{\mathrm{i} m \phi}$.

The complex spectral amplitudes $S_{m}$ are initially set to unity at $m=100$ only, while for all other wavenumbers (within the range of $m=0$ to $m=1024$ ), the amplitudes are zero. The non-zero spectral amplitude is accompanied by a random phase. The latter is applied using the exponential term. The large-scale flow pattern is constructed using

$v_{\phi}=A \cos (\bar{m} \phi)=\frac{A}{2}\left(\mathrm{e}^{\mathrm{i} \bar{m} \phi}+\mathrm{e}^{-\mathrm{i} \bar{m} \phi}\right)$,

where $\bar{m}=20$, defining the wavenumber of the flow component. The amplitude $A$ can be varied to provide the apparent rate at which the small-scale signal is advected.

Substituting Eqs. (5) and (6) into Eq. (4), and normalizing and expressing the changes in $S_{m}$ and $t$ as discrete quantities results in a change in amplitude $\Delta S_{m}$ at a given wavenumber $m$ over a given timestep $\Delta t$. This can be expressed as

$\Delta S_{m}=-\frac{i A}{2}\left[(m-\bar{m}) S_{m-\bar{m}}+(m+\bar{m}) S_{m+\bar{m}}\right] \Delta t$.

By progressing the amplitudes from $S_{m}^{t}$ to $S_{m}^{t+1}$ (i.e., by $\Delta S_{m}$ ) over a timestep $\Delta t$, the supergranule signal at time $t$ is given by

$u_{\phi}^{t}=\sum_{m} S_{m}^{t} \mathrm{e}^{\mathrm{i} m \phi}$.

The timestep and the advection velocity, although arbitrary, were chosen such that the flow pattern changes slowly to yield a smooth evolution with a reasonable temporal resolution. Additionally, the number of timesteps were chosen so that the supergranule pattern would be evolved long enough to observe stretching in some regions and bunching up in others. The longitudinal domain from $\phi=-\pi / 2$ to $\phi=\pi / 2$ was divided into 2048 discrete points to provide the required spatial resolution for the simulation to progress. Rotation was neglected for the sake of simplicity.

Figure 1 shows a series of images sampled at regular timesteps displaying the distribution of the velocity pattern across the domain. It can be seen that as time progresses the pattern evolves. In some regions the pattern is compressed corresponding to the convergent velocities at the boundaries of advecting flow, while in other regions the pattern is stretched out, corresponding to divergent velocities at the centre of the advecting flow. The spacing of the boundaries is coincidental with the wavenumber of the advecting flow in that there exists a boundary every $18^{\circ}$ along the domain. It can be seen that the boundary width continues to reduce as there is no restricting force on the convergent velocities. Its numerical size is of no physical consequence.

This method has also been applied to a convection pattern derived directly from data supplied by MDI (Scherrer et al. 1995). 

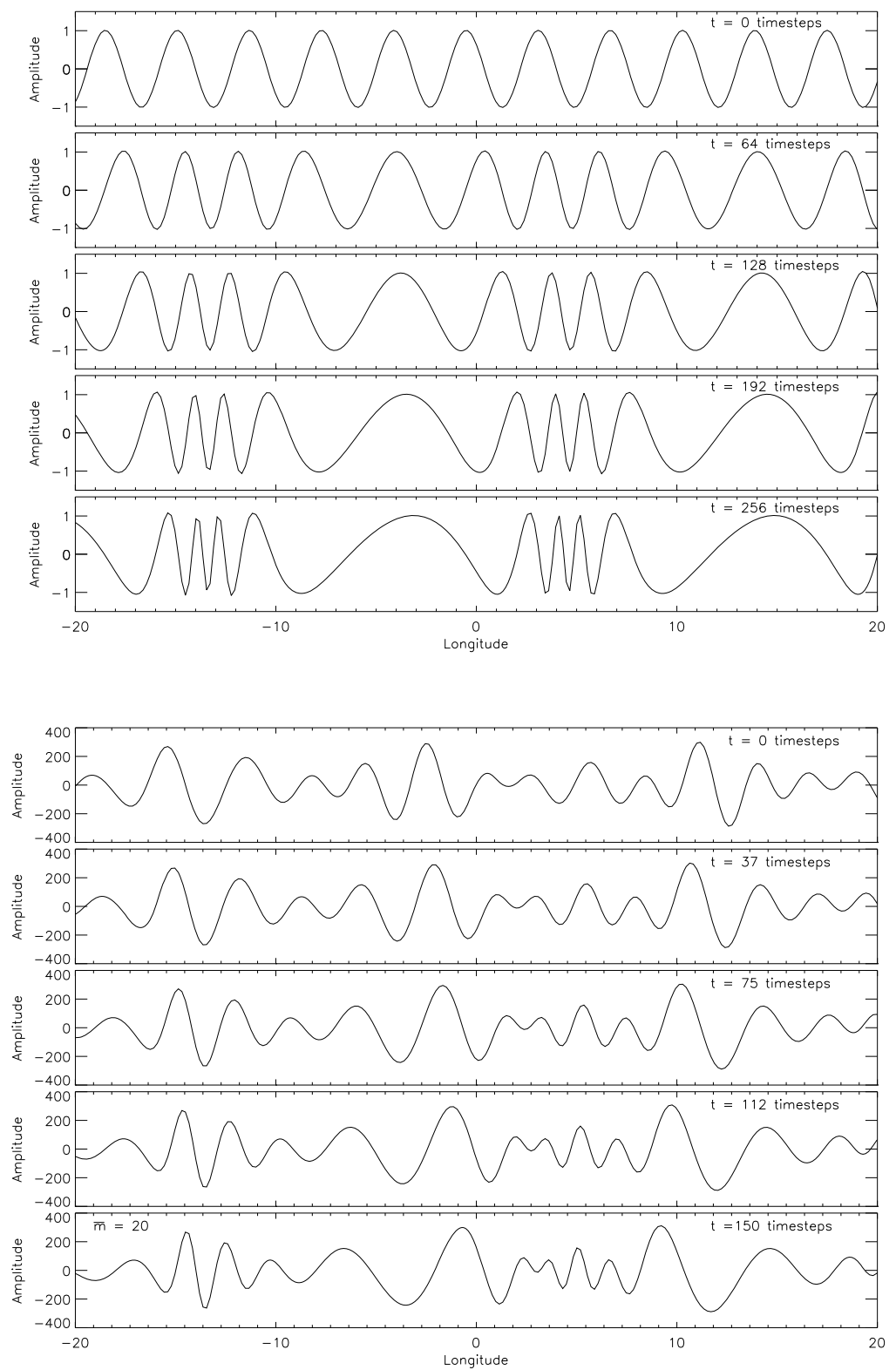

Fig. 1. Time plot series depicting the advection of a sinusoidal pattern by a giant cell component based on a numerical simulation. The plots show a domain of 20 degrees in longitude to either side of the central meridian. Adjacent giant cells exhibit converging flows which bunches up the supergranule pattern, while the centers of the giant cells spread the pattern out. These convergent and divergent flows are prograde or retrograde with respect to any underlying rotational flow that is left out of this simulation for simplicity.
Temporal averages of de-rotated Doppler images that remove $\mathrm{p}$ mode signals from the original data (Hathaway 1988a) are followed by the subtraction of the axisymmetric flows (Hathaway 1992), which yield images that contain signals solely due to convective flows. The images are spectrally filtered in the spatial domain to extract flow-cells that lie within a given wavenumber range (Hathaway et al. 2002). In the present case, the images were filtered with a Gaussian-shaped window function having a $F W H M$ of 128 wavenumbers and centered on $\ell=128$ (Hathaway et al. 2002), a wavenumber near the supergranule peak seen in the convection spectrum (Hathaway et al. 2000). A one-dimensional longitudinal strip (1000 pixels across by 1 pixel in latitude) is extracted near the equator of such a filtered image (May 25, 1996 00:00 UT). The strip containing the Doppler pattern is Fourier transformed in space and the complex spectral amplitudes, $S_{m}$, extracted, each being accompanied by a synthetically produced random phase. The spectral amplitudes are then evolved using the previously described method to simulate the advection of the supergranule pattern by the giant cell component.
Figure 2 shows a series of images similar to that shown in Fig. 1, but using data from a real Doppler image. Although the amplitudes are modulated by the spectral filter, the velocities are still representative of supergranular flows. The top image shows the initial supergranule Doppler pattern, while the subsequent images show the pattern undergoing advection. As in Fig. 1, the giant cell component, with $\bar{m}=20$, produces boundaries of convergent/divergent flows every $18^{\circ}$ along the domain.

The simulation was repeated to model giant cell components situated at other wavenumbers. Figure 3 shows the resulting wavenumber patterns after advection by giant cells of $\bar{m}=10,20$ and 40 , with respectively corresponding boundaries at every $36^{\circ}$, $18^{\circ}$ and $9^{\circ}$ along the domain. Note the correlation between the number of giant cells contained within the domain to the rate of supergranule advection.

\section{Conclusions and outlook}

The main focus of this work is to present a new method for the treatment of the advection of solar supergranulation by giant cells, which is a large-scale analogue to the observation of 


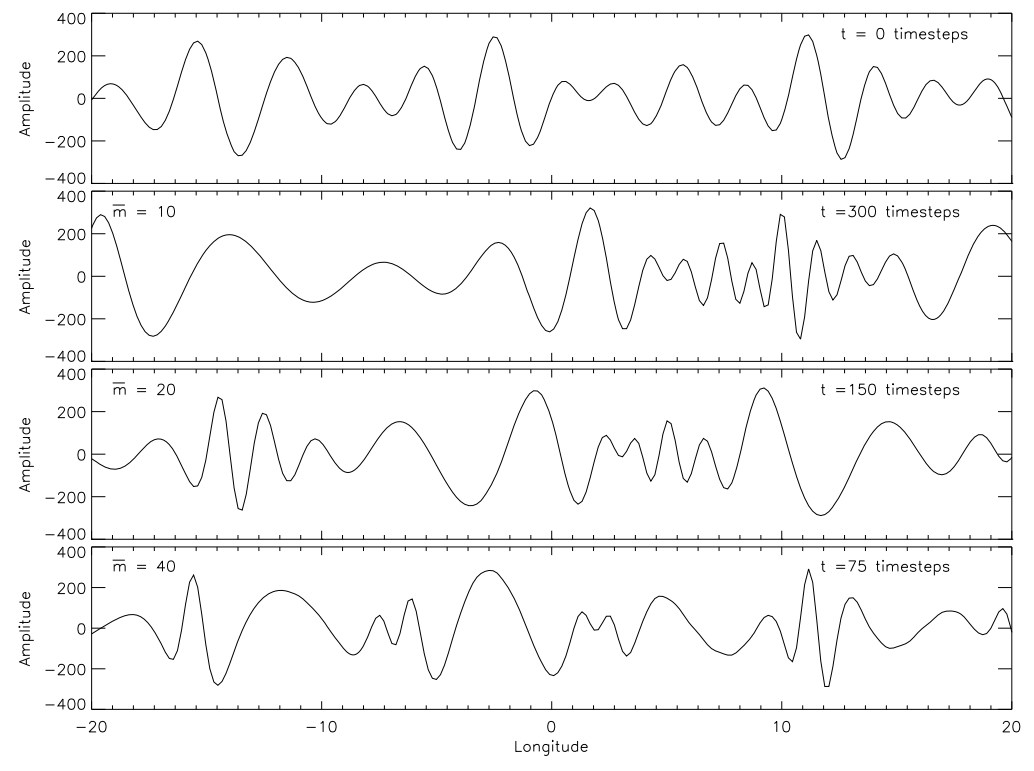

Fig. 3. The top image shows an initial supergranule pattern. Those that follow show the resultant patterns of advection by different sized giant cell components after a particular number of timesteps. granule advection by supergranules (Rieutord et al. 2008). Our underlying goal is to provide the methodology for computing more detailed models that allow the exploration of the effects of a giant cell component on simulated supergranulation patterns in conjunction with empirical data. As a test case, we illustrate the advection of a velocity signal described by a sinusoidal component of a given wavenumber by a large scale flow described by another sinusoidal component with a significantly smaller wavenumber. The wavenumbers of the flows $(m=100$, $\bar{m}=20$ ) were chosen to be representative of supergranules and giant cells, respectively.

We extend our studies to simulate the advection of a real supergranule signal, derived from MDI Dopplergrams, by giant cells. The results show that in some regions of the domain the supergranule signal becomes bunched, whereas in other regions they spread apart. This relates to divergent and convergent flows at the centres and boundaries of giant cells, respectively. Such convergent and divergent flows of giant cells have been derived by studies of the proper motions of Ca II mottles (Schwan \& Wöhl 1978). Note that such signals could be visible at the solar surface either by spectral analysis or correlation tracking. We have also performed these simulations using giant cells of different sizes and find that the advection occurs faster for a domain consisting of smaller giant cells that for larger ones.

Previous spectral analysis of MDI Dopplergrams by Gizon et al. (2003) and Schou (2003) provide observational evidence of prograde and retrograde motions of the supergranule signal along the equator. Additionally, Schou (2003) presents observations that indicate the signal also showing poleward and equatorward motions. He suggests that this is evidence of the wavelike nature of supergranulation. Earlier suggestions of wavelike evidence derived from results by Duvall (1980), Snodgrass \& Ulrich (1990), and Beck \& Schou (2000) seem to have been explained by Hathaway et al. (2006) who were able to attribute the majority of the equatorial signal to a geometric projection effect. However, a remaining signal, including a retrograde component (Gizon et al. 2003), was still unaccounted for. Thus, it may be possible to attribute this remaining signal to the advection of supergranules by giant cells, which will require more detailed studies in the future. Such studies may include simulating the giant cell influence within a two-dimensional domain that would require analysing the coupling between the latitudinal and longitudinal ( $\ell$ and $m$, respectively) in a similar vein to how the advection due to differential rotation has recently been performed Hathaway et al. (2008). Further refinements have to be made to evolve the supergranule pattern over time so that the simulation may essentially run indefinitely.

Acknowledgements. This work was supported by NASA's Office of Space Science through a grant from its Solar and Heliospheric Supporting Research and Technology Program. We also acknowledge valuable discussions with D. H. Hathaway and welcome the comments of an anonymous referee. SOHO is a project of international cooperation between ESA and NASA.

\section{References}

Beck, J. G., \& Schou, J. 2000, Sol. Phys., 193, 333

Beck, J. G., Duvall, Jr., T. L., \& Scherrer, P. H. 1998, Nature, 394, 653

Chandrasekhar, S. 1961, Hydrodynamic and Hydromagnetic Stability (London: Oxford University Press)

Duvall Jr., T. L. 1980, Sol. Phys., 66, 213

Gizon, L., Duvall Jr., T. L., \& Schou, J. 2003, Nature, 421, 43

Hathaway, D. H. 1988a, Sol. Phys., 117, 1

Hathaway, D. H. 1988b, Sol. Phys., 117, 329

Hathaway, D. H. 1992, Sol. Phys., 137, 15

Hathaway, D. H., Beck, J. G., Bogart, R. S., et al. 2000, Sol. Phys., 193, 299

Hathaway, D. H., Beck, J. G., Han, S., \& Raymond, J. 2002, Sol. Phys., 205, 25

Hathaway, D. H., Williams, P. E., \& Cuntz, M. 2006, ApJ, 644, 598

Hathaway, D. H., Williams, P. E., \& Cuntz, M. 2008, in Solar-Stellar

Dynamos as Revealed by Helio- and Asteroseismology, ed. M. Dikpati, I.

Gonzalez-Hernandez, T. Arentoft, \& F. Hill (San Francisco: ASP), ASP Conf. Proc., in press

Kitai, R., Kurokawa, H., Funakoshi, Y., et al. 1999, Adv. Space Res., 24, 237

Küveler, G. 1983, Sol. Phys., 88, 13

Leighton, R. B., Noyes, R. W., \& Simon, G. W. 1962, ApJ, 135, 474

Miesch, M. S., Brun, A. S., DeRosa, M. L., \& Toomre, J. 2007, ApJ, 673, 557

November, L. J., Toomre, J., Gebbie, K. B., \& Simon, G. W. 1981, ApJ, 245, L123

Rast, M. P. 2003, in Local and Global Helioseismology: The Present and Future, ed. H. Sawaya-Lacoste, Noorwijk: ESA, ESA SP-517, 163

Rieutord, M., Meunier, M., Roudier, T., et al. 2008, A\&A, 479, 17

Scherrer, P. H., Bogart, R. S., Bush, R. I., et al. 1995, Sol. Phys., 162, 129

Schou, J. 2003, ApJ, 596, L262

Schwan, H., \& Wöhl, H. 1978, A\&A, 70, 297

Simon, G. W., \& Leighton, R. B. 1964, ApJ, 140, 1120

Simon, G. W., \& Weiss, N. O. 1968, ZAp, 69, 435

Snodgrass, H. B., \& Ulrich, R. K. 1990, ApJ, 351, 309

Stein, R. F., \& Nordlund, A. 1998, ApJ, 499, 914

Strous, L. H., \& Simon, G. W. 1998, in Synoptic Solar Physics, ed. K. S. Balasubramaniam, J. Harvey, \& D. Rabin (San Francisco: ASP), 140, 161 Ulrich, R. K. 2001, ApJ, 560, 466

Williams, P. E., Hathaway, D. H., \& Cuntz, M. 2007, ApJ, 662, L135 